\title{
Experimental overview of spectroscopy from heavy hadron decays
}

\section{Ignacio Bediaga*t†}

CBPF - Centro Brasileiro de Pesquisas Física

E-mail: bediaga@cern. ch

\section{Patricia C. Magalhães}

CBPF - Centro Brasileiro de Pesquisas Física

\begin{abstract}
We present and discuss some experimental approaches involving spectroscopy from heavy meson decays going to light mesons. In particular we emphasis the scalar resonance f0(980), which have different well determined parameter of mass and width obtained from $J / \psi$ and D decays. We will also show how the $\mathrm{CP}$ violation observed in charmless three body decay can be used as tool to understand hadron hadron interaction at low mass region.
\end{abstract}

XVII International Conference on Hadron Spectroscopy and Structure - Hadron2017

25-29 September, 2017

University of Salamanca, Salamanca, Spain

\footnotetext{
*Speaker.

${ }^{\dagger}$ this work of was supported by FAPERG and CNPq Grant No. 157857/2015- 8A.
} 


\section{Introduction}

Heavy hadron weak decays, mainly non-leptonic ones, have been used extensively to study light meson spectroscopy in a complementary way to the usual hadron hadron interactions. These decays have a defined initial spin parity quantum numbers that allowed a clear signature to determine the spin of the intermediary state resonances. Each two body resonance has a particular distribution in the Dalitz plot associated to their particular angular momentum quantum number. Beside that, the interference between well known resonances and other amplitude in a particular decay allows the observation of new resonances states, even if they give a small contributions to the total amplitude, due the interference pattern between them.

However there are many issues related to a multi-body final state interactions (FSI) description of heavy hadron decays that are present on the experimental amplitude analysis on these processes: the quasi-to-body approach of the FSI which neglect bachelor particle participation, the Breit-Wigner parameters for mass and width, the unknown non-resonant amplitudes, and the isobar coefficient for magnitude and phase of each individual amplitude, which are constant along the phase space.

Beside hadron spectroscopy, more recently heavy meson three-body decays has been used as a tool to search and study CP asymmetry $[1,2,4,5,6]$. In charm meson decays the observation of $\mathrm{CP}$ asymmetry would be a hint of new physics, whereas in charged charmless B decays observation indicate a expressive CP asymmetry located in some regions of the Dalitz plot. Moreover, the study of the $\mathrm{CP}$ asymmetry distribution in these three-body phase space have been used in one side to understand the mechanism of the $\mathrm{CP}$ asymmetry production and on other side to understand the dynamics of hadron interactions inside a three-body environment $[5,6,7,8,9]$.

In this paper is organized to present in section two an overview of the experimental features and problems related to light resonance spectroscopy from charm meson decays, focusing on the scalar $f_{0}(980)$ scalar resonance. In section three we present the perspective and inquiring of charmless $\mathrm{B}$ meson decay experiments to study light hadron spectroscopy through the large CP asymmetry distribution observed in these decays. In section four we present our final considerations.

\section{Overview on the experimental light resonances spectroscopy from charm meson decays.}

The scalar $f_{0}(980)$ resonance is one of the most universal particle present in these decays, it has a clear signature in all experimental decays involving two pions or two kaons in the final state. We present an overview of experimental results involving two different initial states: one from charmonium $J / \psi$ decays, the other from weak decays of $\mathrm{D}$ and $\mathrm{B}$ mesons.

\subsection{The $f_{0}(980)$ mass and width parameters from $J / \psi$ decays.}

The MarkII[10] collaboration made an observation of the $f_{0}(980)$ parameters from a inclusive $J / \psi \pi^{-} \pi^{+} X$ decays. They used a Flatté-Breit-Wigner amplitude distribution to fit the $\pi \pi$ mass spectrum:

$$
B W_{f_{0}(980)}=\frac{1}{m_{\pi \pi}^{2}-m_{0}^{2}+i m_{0}\left(\Gamma_{\pi}+\Gamma_{K}\right)}
$$




$$
\begin{aligned}
& \Gamma_{\pi}=g_{\pi} \sqrt{m_{\pi \pi}^{2} / 4-m_{\pi}^{2}}, \\
& \Gamma_{K}=\frac{g_{K}}{2}\left(\sqrt{m_{\pi \pi}^{2} / 4-m_{K}^{2}}+\sqrt{m_{\pi \pi}^{2} / 4-m_{K_{0}}^{2}} .\right.
\end{aligned}
$$

To perform the fit, they fixed $g_{k}=0.2$ and get $m_{0}=956 \pm 6$ and $g_{\pi}=0.088 \pm 0.029$ with the $3700 \pm 700$ observed events.

A more complex and complete analysis was performed by BES Collaboration [11] using the exclusives $J / \psi \rightarrow K^{-} K^{+} \pi^{+} \pi^{-}$and $J / \psi \rightarrow K^{-} K^{+} K^{-} K^{+}$channels. They selected events with two kaons in the $\phi(1020)$ mass region and inspect what remained in $K^{-} K^{+}$and $\pi^{+} \pi^{-}$spectrum. With a partial amplitude analysis they fitted simultaneously the $J / \psi \rightarrow \phi \pi^{-} \pi^{+}$and $J / \psi \rightarrow \phi K^{-} K^{+}$ channels. They constrained the resonance masses and widths Breit-Wigners parameters to be the same in both decays for the resonances amplitudes: $\sigma, f_{2}(1270), f_{0}(1370), f_{0}(1500), f_{2}(1525)$ and $f_{0}(1710)$. The $f_{0}(980)$ was fitted with a Flatté-Breit-Wigner, Eq.(2.1). Other than got the $f_{0}(980)$ Flatté parameters, they floating also the $f_{2}(1270), f_{0}(1370), f_{2}(1525), f_{0}(1790)$ mass and width parameters.

The final adjust for the couple channel partial amplitude analysis results in the following FlattéBreit-Wigner $f_{0}(980)$ parameters : $m_{0}=965 \pm 10 \pm 15 \mathrm{MeV}$ and $g_{K} / g_{\pi}=4.21 \pm 0.25 \pm 0.21$. These $f_{0}(980)$ parameters have been used as an input parameters for other analysis as one can see on literature.

More recently BESIII collaboration presented a quite different result for the $f_{0}(980)$ parameters from the $J / \psi \rightarrow \gamma 3 \pi$ decay. In Fig. 1 there is a clear peak in the $3 \pi$ invariant mass, associated to the $\eta(1405)$. From the $J / \psi \rightarrow \gamma \eta(1405)$ events, they look at $\pi^{-} \pi^{+}$invariant mass and observed a clear $f_{0}(980)$ signal, much narrow than the other experimental results for this scalar resonance. The width parameter measured was $9.5 \pm 1.1 \mathrm{MeV}$ and mass $989.9 \pm 0.4 \mathrm{MeV}$, these values were obtained with a regular Breit-Wigner.

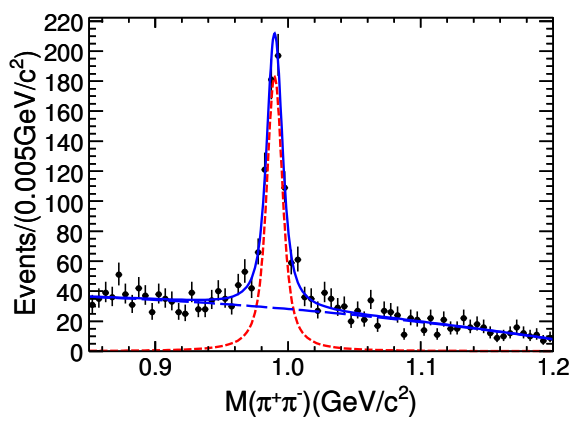

Figure 1: The $\pi^{+} \pi^{-}$invariant mass spectra with $\pi^{+} \pi^{-} \pi^{0}$ in the $\eta(1405)$ mass region. The solid curve is the result of the fit the dotted curve is the $f_{0}(980)$ signal and dashed one denotes the background polynomial.

The non isospin conservation of this decay, has been attributed as the responsible of this apparent anomaly on the width of the scalar $f_{0}(980)$ resonance [13]. A similar decay $J / \psi \rightarrow \phi \pi^{0} \pi^{-} \pi^{+}$, also observed by BESIII collaboration[14] with a isospin violation, confirm this narrow $f_{0}(980)$ resonance. Direct measurement of the Breit-Wigner parameters, gave $m_{0}=989.4 \pm 1.3$ and $\Gamma=$ $15.3 \pm 4.7 \mathrm{MeV}$, compatible with the previous values from $\eta(1405) \rightarrow f_{0}(980) \pi^{0}$. 


\subsection{The $f_{0}(980)$ mass and width parameters from charm meson weak decays}

Several three body $\mathrm{D}$ weak decays were used to study the $f_{0}(980)$ parameters. The pioneer in these studies was the E791 collaboration in $D_{s}^{+} \rightarrow \pi^{+} \pi^{-} \pi^{+}$decay[15]. To performed this study they introduced in the Isobaric amplitude Model the possibility to float mass and width of an resonance amplitude. The $f_{0}(980) \pi^{+}$amplitude has approximately half of the $D_{s}^{+} \rightarrow \pi^{+} \pi^{-} \pi^{+}$events with a clear signature in the middle of the Dalitz plot. This allows E791 even with low statistic, to perform a precise estimative of $f_{0}(980)$ parameters using the Flatté-Breit-Wigner parametrization: $m_{0}=977 \pm 3 \pm 0.01 \mathrm{MeV}, g_{\pi}=0.08 \pm 0.01 \pm 0.01$ and $g_{K}=0.02 \pm 0.04 \pm 0.03$. One can note that the $g_{K}$ value is compatible with zero in a clear opposition to the value obtained by BES from $J \psi$ decays[11]. By imposing $g_{K}=0$ in their analyses, E791 collaboration[15] found the width parameter to be $\Gamma=44 \pm 3 \pm 2 \mathrm{MeV}$.

This result together with other involving the scalar particles sigma and the kappa, observed respectively in the $D^{ \pm} \rightarrow \pi^{ \pm} \pi^{+} \pi^{-}[16]$ and $D^{ \pm} \rightarrow K^{ \pm} \pi^{+} \pi^{-}$[17] by the same E791 experiment, open an important and long discussion related to the rule of the bachelor particle in the three body FSI. In short, the common believe on hadronic three-body decays was that the bachelor particle should be a simple spectator to the other two hadron interactions and consequently the final amplitude must follow the Watson theorem[18] and the phase variation, along the invariant mass of the pair in charm three body phase space, should be the same as the one observed in elastic scattering experiments. However, as we show bellow, this do not correspond to was observed.

In order to test this hypothesis, E791 collaboration redo the $D^{+} \rightarrow K^{-} \pi^{+} \pi^{+}$analysis, with the same data sample, using a Model Independent Partial Wave Analysis (MIPWA) [19], replacing the scalar resonances kappa and $K_{0}^{*}(1430)$ Breit-Wigners, by a continuous S-wave parametriztion $a\left(m_{K^{-} \pi^{+}}\right) e^{-i \phi\left(m_{K^{-}} \pi^{+}\right)}$, dividing the Dalitz plane in rectangular bins. For the spin one and two resonances, it was used the regular Isobar Model, in the same way used on the previous analysis. In Fig. 2 (left) one can see the results for the phase in the S-wave obtained by this analysis compared with the S-wave phase distribution obtained by scattering amplitude[20]. As we can see from Fig.2

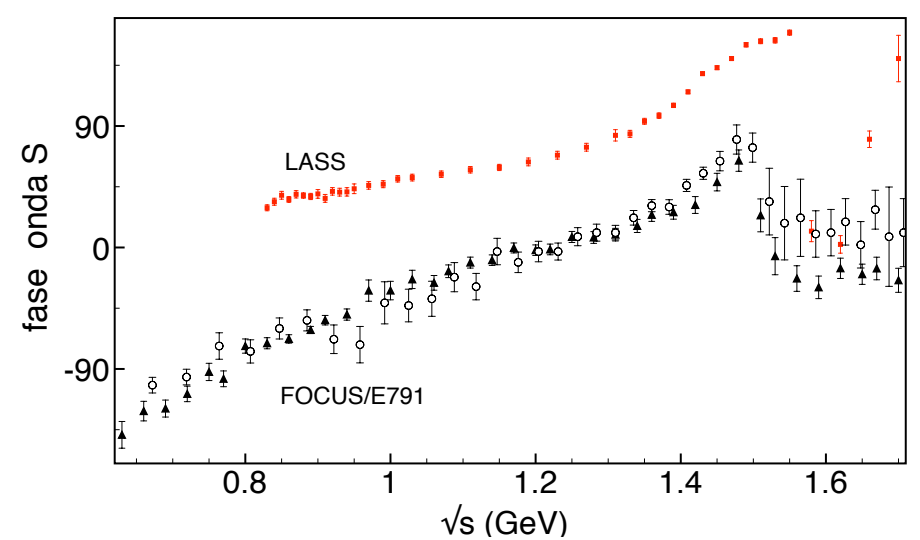

Figure 2: S-wave phase of the $K^{-} \pi^{+}$pair: obtained by FOCUS[21](triangle) and E791[17] (circle) collaborations in $D^{+} \rightarrow K^{-} \pi^{+} \pi^{+}$decay; and obtained by LASS[20] in the free $K^{-} \pi^{+}$scattering.

there are an overall difference and also a different dependence in $K^{-} \pi^{+}$invariant mass between the phases, specially at low mass, showing that Watson theorem[18] does not work properly in this 
decay. FOCUS[21] collaboration performed similar analysis, with high statistic and got similar behaviour for the S-wave phase motion obtained by E791.

There are some important theoretical works on $D^{+} \rightarrow K^{-} \pi^{+} \pi^{+}$[22, 23, 24, 25]. Although based on different frameworks all of them show the importance of three-body FSI to describe the experimental data. In particular, Refs. [22, 23] have showed that hadron loops introduce new complex structures to the $D^{+} \rightarrow K^{-} \pi^{+} \pi^{+}$amplitude which modify both the S- and P-wave phase and succeeded in explaining the observed discrepancy presented in Fig.2 (left).

More recently, BaBar collaboration applied the MIPWA technique to the $D_{s}^{+} \rightarrow \pi^{+} \pi^{-} \pi^{+}$ decay[26]. The scalar amplitude extracted from this analysis was compared with the E791[15] with one order of magnitude less events. There is a good agreement between them in the $f_{0}(980)$ mass region but not on the entire phase space, being worse to values of $\pi^{-} \pi^{+}$mass square bigger than $1.1 \mathrm{GeV}^{2}$. The scalar phase motion obtained with the MIPWA was also compared with the pion pion elastic scattering CERN-Munich experiment[27] and it is presented in Figure $3^{1}$. Similar

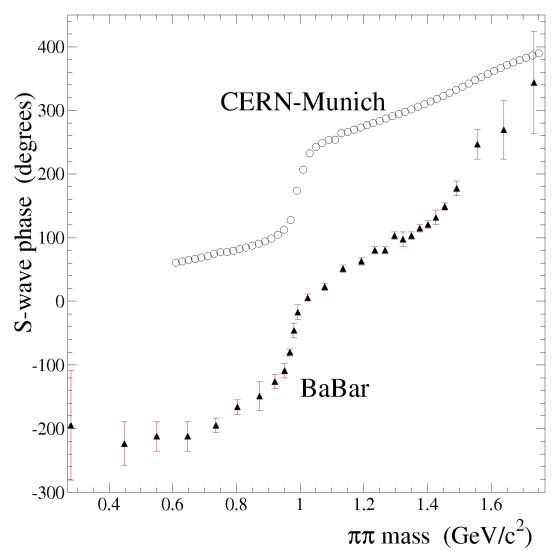

Figure 3: S-wave phase of the $\pi^{-} \pi^{+}$pair: obtained by Babar [26] collaboration in $D_{s}^{+} \rightarrow \pi^{+} \pi^{-} \pi^{+}$decay; and obtained by CERN-Munich[27] in the free $\pi^{-} \pi^{+}$scattering .

to what was observed in the $D^{ \pm} \rightarrow K^{-} \pi^{+} \pi^{ \pm}$decay, Fig. 2 (right) shows that there is an overall difference of about 200 degrees in $\pi^{+} \pi^{-}$invariant mass between the pion pion elastic scattering observed by CERN-Munich experiment[27] and the observed by the BaBar to $D_{s}^{+} \rightarrow \pi^{+} \pi^{-} \pi^{+}$ decay. Moreover, we can see that the phase motion has a different behavior around the $f_{0}(980)$ mass region. Therefore, it is natural that they have a different Breit-Wigner mass and width parameters as it was pointed out by $D_{s}^{+} \rightarrow \pi^{+} \pi^{-} \pi^{+}$E791 result.

BaBar collaboration have other important result in what concerns $f_{0}(980)$ spectroscopy in charm three-body decay. They applied the MIPWA to the $D_{s}^{+} \rightarrow K^{+} K^{-} \pi^{+}$decay, with high statistic[29], to investigate the scalar $K^{+} K^{-}$invariant mass. The result[29] shows a smooth phase variation from threshold to $1.15 \mathrm{GeV}$, which is complete different from the $D_{s}^{+} \rightarrow \pi^{+} \pi^{-} \pi^{+}$decay obtained by the same experimental collaboration. Once this analysis was insensible to the to the coupling of the with the $\pi^{+} \pi^{-}$channel, they used a regular Breit-Wigner distribution to

\footnotetext{
${ }^{1}$ want to thanks to Alberto dos Reis for this Figure and discussions on that matter.
} 
$f_{0}(980)$ and obtain from their analysis a mass parameter of $m_{0}=922 \pm 0.003 \mathrm{MeV}$ and the width $\Gamma=240 \pm 80 \mathrm{MeV}$. It is important to point out the these two results from the same experimental collaboration and same initial state, confirm the importance of the environment to modify the mass and width parameters of a Breit-Wigner like distribution.

In conclusion, for this section, the $f_{0}(980)$ Breit-Wigner mass and width parameters measured seems to depend strongly on the physics production process involving decays of heavy mesons. Moreover, analysis performed in charm three-body D decay showed that FSI are important and Watson theorem can not be apply properly to these three-body decays. The difference between of the phase motion in the elastic scattering to these decays process, have been attributed to the participation of the bachelor particle on the total interaction. Consequently, if there are a phase motion different from the scattering, in a resonance region, is natural that the Breit-Wigner parameters change from one process to the other.

\section{Perspective of CP violation studies in charmless three body B meson decay}

The huge phase space difference between charmless three-body B meson decays and charm meson three -body decay rise some questions yet without a clear responses[28] Does the nonresonant remain flat? How does it looks like? Does the two body magnitudes and phases are the same in all phase space? Does the $(2+1)$ approximation works in these decays? Can the entire phase space be described for a single formalism?

In order to answer these and other questions it would be necessary two crucial elements: a high statistic samples of these decays and a strong collaboration between experimental and theoretical physicists. LHCb collaboration already started both. In fact, $B^{ \pm} \rightarrow K^{-} K^{+} K^{ \pm}$and $B^{ \pm} \rightarrow K^{ \pm} \pi^{+} \pi^{-}$ decays have already a hundred and about two hundred signal events respectively, available in run 1 and probably more than three time this statistics from run 2. LHCb collaboration has been also stimulating some meetings between experimental and theoretical physicists in different subjects, but in particular, on the multibody hadron interaction. Some presentations can be found in the "annual LHCb series: Implications of LHCb measurements and future prospects" and a dedicated workshop on this subjects in Rio de Janeiro[30].

There are though some important feature that we have learned from previous Babar, Belle and, more recently, $\mathrm{LHCb}$ collaborations. One important thing is that at low mass the two-body resonances are still dominating the total B decay amplitude contributions: rho $(770), K^{*}(890)$, $\phi(1020)$ and $f_{0}(980)$, among others; like in charm three-body decays. However, differently from charm decays, the nonresonant contribution in B decays in an important contribution in all channels and also, the distribution of this amplitude should not be flat, like it is represented in charm decays. Although it is not clear how exactly it looks like and what are the mechanism that generate them.

Charged charmless B three-meson decays has been presenting a significant inclusive CP violation on data[5]. Those decays present a rich CP distribution structure in Dalitz plot, with some regions with a high positive $\mathrm{CP}$ asymmetry nearby regions also with this kind of asymmetry but with opposite signal as we can see in the figure below. Note that the Fig4 is a result of the subtraction of the negative B meson Dalitz plot for the positive one. The color shows the range of the $\mathrm{CP}$ asymmetry. 

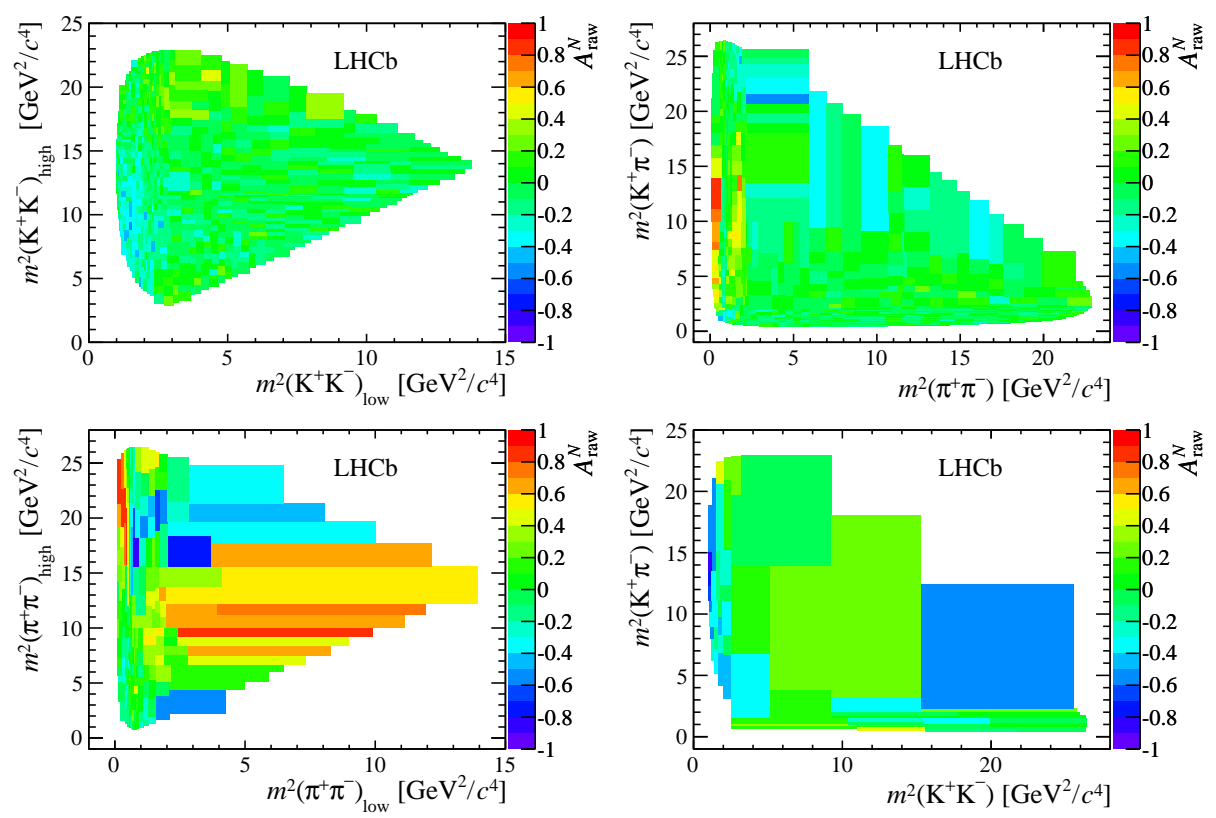

Figure 4: $\mathrm{LHCb}$ results on direct CP asymmetry for the channels: $B^{ \pm} \rightarrow K^{ \pm} \pi^{+} \pi^{-}, B^{ \pm} \rightarrow K^{ \pm} K^{+} \pi^{-}$, $B^{ \pm} \rightarrow \pi^{ \pm} \pi^{+} \pi^{-}$and $B^{ \pm} \rightarrow K^{ \pm} K^{+} K^{-}$.

In order to produce $\mathrm{CP}$ asymmetry the amplitudes must have at least two components and these components must have different weak $(\delta)$ and strong phases $(\phi)$, as given generically by Eqs.(3.1) and (3.2). The value of this asymmetry is proportional to the results of Eq.(3.3)

$$
\begin{aligned}
A(B \rightarrow f) & =A_{1} e^{i\left(\delta_{1}+\phi_{1}\right)}+A_{2} e^{i\left(\delta_{2}+\phi_{2}\right)} \\
A(\bar{B} \rightarrow \bar{f}) & =A_{1} e^{i\left(\delta_{1}-\phi_{1}\right)}+A_{2} e^{i\left(\delta_{2}-\phi_{2}\right)} \\
\left|A_{B \rightarrow f}\right|^{2}-\left|A_{\bar{B} \rightarrow \bar{f}}\right|^{2} & =-4 A_{1} A_{2} \sin \left(\delta_{1}-\delta_{2}\right) \sin \left(\phi_{1}-\phi_{2}\right) .
\end{aligned}
$$

In general, charmless B decays has a tree diagram with a weak phase $\gamma$ from CKM matrix; together with a penguin diagram with a quark anti-quart loop. Although the penguin loop is the most common source of strong phase considered in these decays, there could be other sources in the hadronic FSI. Important strong phase difference components can be generated in these decays though rescattering, Breit-Wigners and the interference between two different partial waves.

The weak phase signal is defined positive to the particle and negative to the anti-particle, so it is not responsible to the observed CP violation signal changing in the three body B decays. Consequently the high $\mathrm{CP}$ violation variation observed in Dalitz plane necessarily came from a strong phase variation, allowing a clear signature of this behaviour along the phase space.

It possible to identify two sources of strong phase differences in charmless three body charged $\mathrm{B}$ decays in $\mathrm{LHCb}$ data[5] that can be responsible for the $\mathrm{CP}$ asymmetry. One is related to the $\pi^{+} \pi^{-} \rightarrow K^{+} K^{-}$rescattering, and other to the interference between $\mathrm{S}$ and $\mathrm{P}$ wave at low $\pi^{+} \pi^{-}$ invariant mass. Actually they showed a clear correlation between the channels $B^{ \pm} \rightarrow K^{ \pm} \pi^{+} \pi^{-}$ and $B^{ \pm} \rightarrow K^{ \pm} K^{+} K^{-}$decays, observed in the region where $\pi^{+} \pi^{-} \rightarrow K^{+} K^{-}$has an important contribution in the hadron-hadron scattering amplitude [31] - i.e. between 1 and $1.6 \mathrm{GeV}$. The 
$B^{ \pm} \rightarrow K^{ \pm} \pi^{+} \pi^{-}$has a positive $\mathrm{CP}$ asymmetry in this region whereas the $B^{ \pm} \rightarrow K^{ \pm} K^{+} K^{-}$has a negative one. A similar correlation in the $\mathrm{CP}$ asymmetry, i.e. in the same mass region, was observed between the two channels $B^{ \pm} \rightarrow \pi^{ \pm} K^{+} K^{-}$and $B^{ \pm} \rightarrow \pi^{ \pm} \pi^{+} \pi^{-}$. These results indicate that the re-scattering process $\pi^{+} \pi^{-} \rightarrow K^{+} K^{-}$is present in these decays [7, 8], carrying the strong phase necessary for CP violation and conserving CPT global symmetry as discussed in Ref. [7, 8].

Another source of CP violation identified in these data for the low $\pi^{+} \pi^{-}$invariant mass in the $B^{ \pm} \rightarrow K^{ \pm} \pi^{+} \pi^{-}$and $B^{ \pm} \rightarrow \pi^{ \pm} \pi^{+} \pi^{-}$decays is related to the interference between $\mathrm{S}$ and $\mathrm{P}$ wave projections. Figure 5 shows the $\pi^{+} \pi^{-}$spectrum for $B^{-} \rightarrow \pi^{-} \pi^{+} \pi^{-}$(open triangle) and
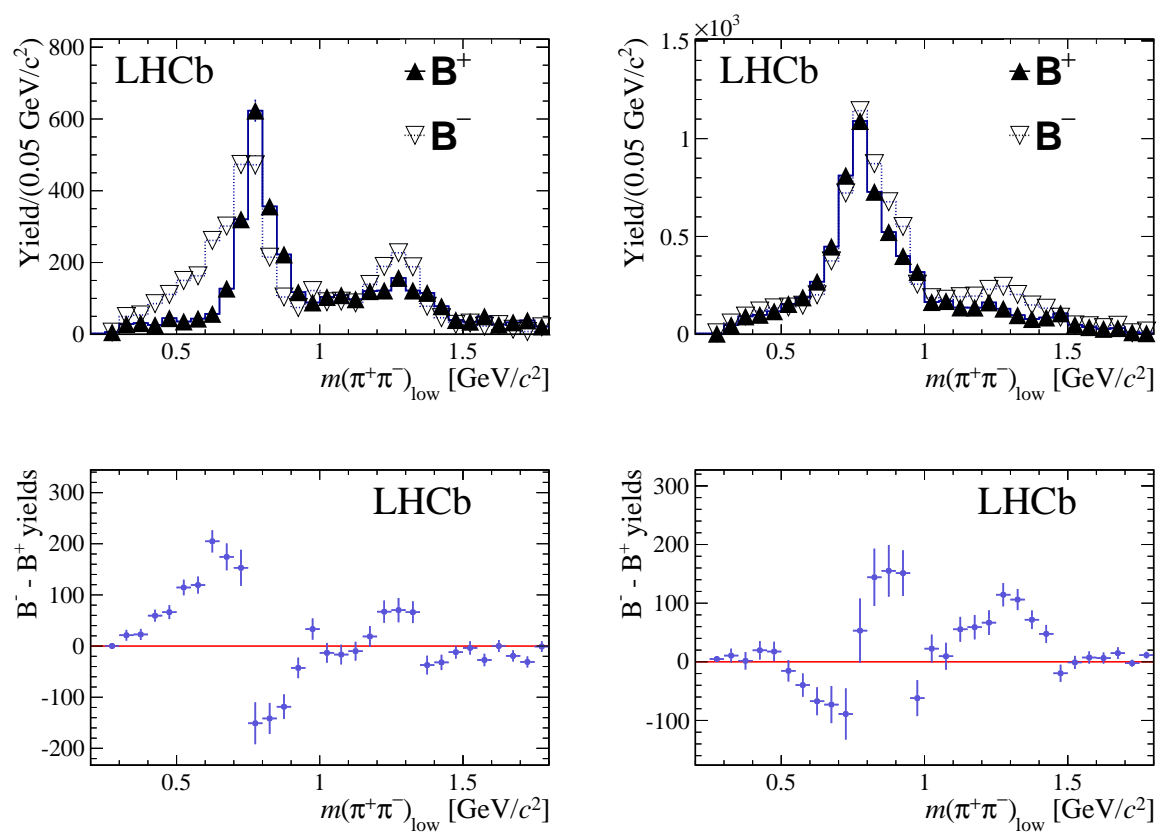

Figure 5: Projections in bins of the $m(\pi \pi)_{l o w}$ variable of $(\mathrm{a}, \mathrm{b})$ the number of $B^{-}$and $B^{+}$signal events and (c, d) their difference for $B^{ \pm} \rightarrow \pi^{+} \pi^{-} \pi^{ \pm}$decays. The plots are restricted to events with (a, c) $\cos \theta<0$ and $(b, d) \cos \theta>0$, with $\cos \theta$ defined in the text. The yields are acceptance-corrected and backgroundsubtracted. A guide line for zero (horizontal red line) was included on plots (c, d).

$B^{+} \rightarrow \pi^{+} \pi^{+} \pi^{-}$(full triangle) decays, in two different regions of the Dalitz plot, defined by the vector angular distribution $\cos \theta$. One for $\cos \theta>0$ Fig. 5(top-left) and another for $\cos \theta<0$ Fig. 5 (top-right). On the bottom part of these Figures we could find the spectrum subtraction. One can see a zero in both distributions at the $\rho^{0}(770)$ mass distribution position, indicating that the interference term between a S-wave and vector $\rho^{0}(770)$ is dominated by the real term of the Breit-Wigner. The signal exchange between Fig.5(top-left) and Fig.5(top-right) shows that this interference term is proportional to the $\cos \theta$, which vary between -1 to 1 in the spectrum, as one can expect from a $\mathrm{S}$ and $\mathrm{P}$ wave interference. A similar process can be seen in $\pi^{+} \pi^{-}$low mass spectrum of the $B^{ \pm} \rightarrow K^{ \pm} \pi^{+} \pi^{-}$decay.

$\mathrm{LHCb}$ data[5] also present $\mathrm{CP}$ asymmetries placed in high mass regions of the Dalitz plane, near the open channel of charm mesons. In Fig. 6 we access the amount of LHCb [32] events related to CP violation in the four channels $B^{ \pm} \rightarrow h^{ \pm} h^{+} h^{-}$obtained by the subtraction of events 

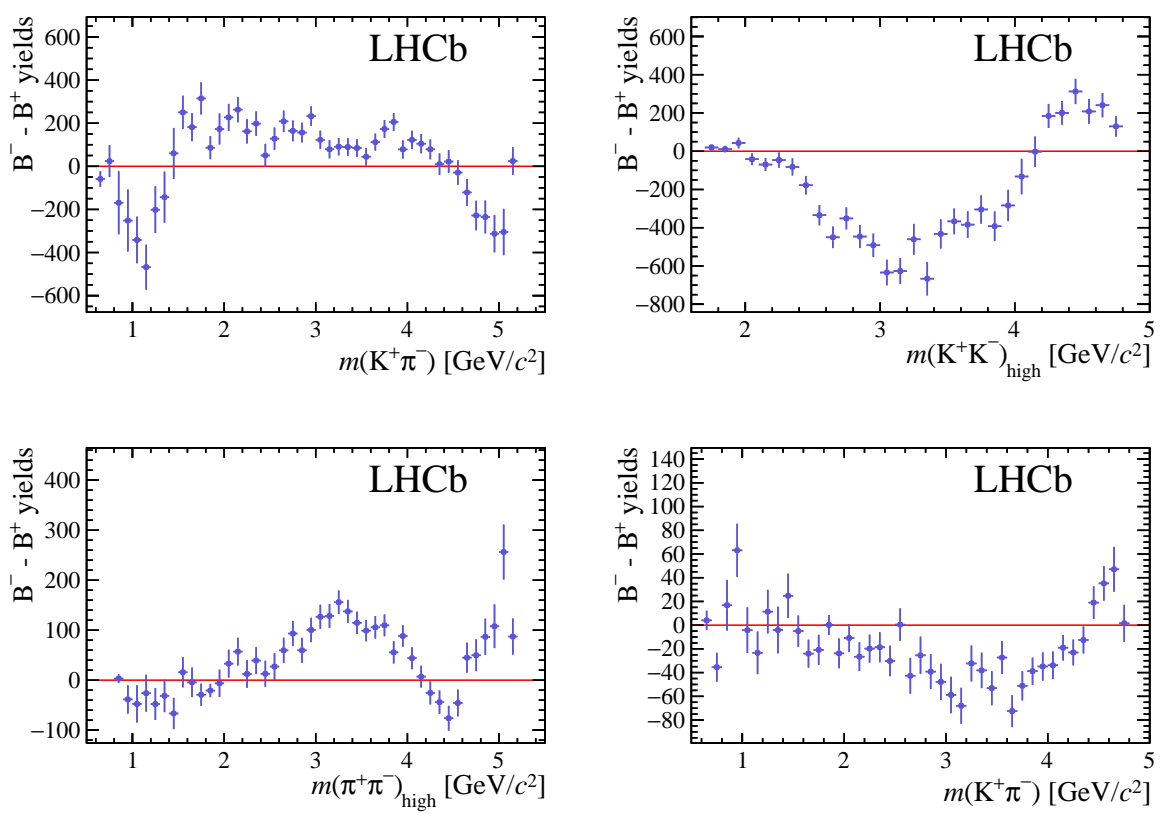

Figure 6: $\mathrm{CP}$ asymmetry projection on $m(h h)$ (similar to the previous figure for all phase -space) for the the channels: $B^{ \pm} \rightarrow K^{ \pm} \pi^{+} \pi^{-}$(top-left), $B^{ \pm} \rightarrow K^{ \pm} K^{+} K^{-}$(top- right), $B^{ \pm} \rightarrow \pi^{ \pm} \pi^{+} \pi^{-}$(bottom- left ), $B^{ \pm} \rightarrow \pi^{ \pm} K^{+} K^{-}$(bottom- right).

for $B^{+}$and $B^{-}$integrated in $m(h h)$. On can see from Fig 6 that in all channels the CP asymmetry changes sign crossing zero at $\approx 4 \mathrm{GeV}$. Moreover, it is possible to observes a correlation between the two top and bottom graphs: they present an opposite direction of CP asymmetry sign change. Inspired by the $\pi \pi \rightarrow K K$ rescattering and by the CP sign change near the $D \bar{D}$ open channel, we investigate in a recent paper[33] the possibility of the double charm rescattering to light mesons to be a source of a new strong phase variation at high mass that could be a new mechanism of $\mathrm{CP}$ violation on those decays.

\section{Final remarks}

Three body charm meson decays have been given many important contribution for light meson spectroscopy, mainly to understand scalar resonances. With the order of millions of charm decays events per channel, LHCb experiment can go further and look at double Cabibbo decays with detail other than redo interesting decays like $D_{s}^{ \pm} \rightarrow \pi^{ \pm} \pi^{+} \pi^{-}$to confirm previous results.

On the other hand, charmless three body B decays already have channels with high statistics, but with many open question raised due to the huge phase space. Thus we have to learn with data and new theoretical inputs. LHCb experiment already have $3 f t^{-1}$ in run 2, collected with $13 \mathrm{TeV}$ in the center of mass energy. At this energy, the $b \bar{b}$ cross section is about twice the run 1[34]. Therefore, we can expect soon a LHCb experimental results with around half million events to $B^{ \pm} \rightarrow K^{ \pm} \pi^{+} \pi^{-}$decay. 
With this statistics and the big amount of CP violation observed in charmless three body B meson decays, we can go deeply to understand many issues involving strong phases along the phase space of these decays. Also understand better the necessary tools and theoretical issues to perform a realistic amplitude analysis, necessary to extract physical quantities.

\section{References}

[1] I. Bediaga, I.I. Bigi, A. Gomes, G. Guerrer, J. Miranda, A.C.dos Reis. Phys.Rev. D80 (2009) 096006.

[2] I. Bediaga, J. Miranda, A.C. dos Reis, I.I. Bigi, A. Gomes, J.M. Otalora Goicochea, A. Veiga. Phys.Rev. D86 (2012) 036005.

[3] I. Bediaga, I.I. Bigi, J. Miranda, A. Reis. Phys.Rev. D89 (2014) no.7, 074024.

[4] LHCb Collaboration (R. Aaij et al.) Phys. Lett. B728, 585 (2014).

[5] LHCb Collaboration (Roel Aaij et al.). Phys.Rev. D90 (2014) no.11, 112004, Phys.Rev.Lett. 112 (2014) no.1, 011801 and Phys.Rev.Lett. 111 (2013) 101801.

[6] LHCb Collaboration (Roel Aaij et al.). Phys.Rev.Lett. 113 (2014) no.14, 141801.

[7] I. Bediaga, T. Frederico, and O. Lourenço, Phys. Rev. D 89 no.9 (2014) 094013

[8] J. H. Alvarenga Nogueira, I. Bediaga, A. B. R. Cavalcante, T. Frederico and O. Lourenço, Phys. Rev. D 92 no.5 (2015) 054010.

[9] J. H. Alvarenga Nogueira, I. Bediaga, T. Frederico, P.C. Magalhães, J. Molina Rodriguez. Phys.Rev. D94 (2016) no.5, 054028.

[10] MarkII collaboration (G. Gidal et al.) Phys.Lett. 107B (1981) 153-158.

[11] BES Collaboration (M. Ablikim et al.). Phys.Lett. B607 (2005) 243-253.

[12] BESIII Collaboration (M. Ablikim et al.). Phys.Rev.Lett. 108 (2012) 182001.

[13] F. Aceti, W. H. Liang, E. Oset, J. J. Wu and B. S. Zou, Phys. Rev. D 86, 114007 (2012),F. Aceti, J. M. Dias and E. Oset, Eur. Phys. J. A 51, no. 4, 48 (2015), S. Sakai, E. Oset and W. H. Liang, arXiv:1707.02236 [hep-ph].

[14] BESIII Collaboration (M. Ablikim et al.). Phys.Rev. D92 (2015) no.1, 012007.

[15] E791 Collaboration, Phys.Rev.Lett. 86 (2001) 765.

[16] E.M. Aitala et al. (E791), Phys. Rev. Lett. 86770 (2001); 86765 (2001).

[17] E.M. Aitala et al. (E791), Phys. Rev. Lett. 89, 121801 (2002).

[18] K.M. Watson, Phys.Rev. 88 (1952) 1163.

[19] E791 Collaboration, E791 Collaboration (E.M. Aitala et al.). Phys.Rev. D73 (2006) 032004, Erratum: Phys.Rev. D74 (2006) 059901.

[20] D. Aston et al., Nucl.Phys. B 296, 493 (1988); P. Estabrooks et al., Nucl. Phys. B 133, 490 (1978).

[21] J.M. Link et al. [FOCUS Collaboration], Phys. Lett. B 681, 14 (2009).

[22] Magalhaes, P.C. and Robilotta, M.R. and Guimaraes, K.S.F.F. and others", Phys.Rev. D84 (2011) 094001.

[23] P. C. Magalhães and M.R. Robilotta, M.R., Phys. Rev. D92 9 (2015) 094005. 
[24] S. X. Nakamura, Phys. Rev. D93 1 (2016) 014005.

[25] Niecknig, Franz and Kubis, Bastian, JHEP 10 (2015) 142.

[26] BaBar Collaboration (Bernard Aubert et al.). Phys.Rev. D79 (2009) 032003.

[27] G. Grayer (CERN) et al., Nucl.Phys. B75 (1974) 189.

[28] Ignacio Bediaga, [arXiv:1104.0694], published at: "Alberto Santoro: A Life of Achievements. A Festschrift on the Occasion of his 70th Birthday".

[29] BaBar Collaboration (P. del Amo Sanchez et al.). Phys.Rev. D83 (2011) 052001.

[30] J.H. Alvarenga Nogueira et.al. [arXiv:1605.03889].

[31] D. H. Cohen et.al. Phys.Rev. D22(1980) 2595.

[32] Additional information from Ref[12] https://cds.cern.ch/record/1751517/files/.

[33] I. Bediaga, T. Frederico, and P.C. Magalhães, ArXiV:1712.01230 and in the proceeding of this conference.

[34] LHCb Collaboration (Roel Aaij et al.), Phys.Rev.Lett. 118 (2017) no.5, 052002, Erratum: Phys.Rev.Lett. 119 (2017) no.16, 169901.? 